\title{
The Theoretical Derivation of a One-sided and Two-sided $P$ Value Calculation Formula of One-dimensional Continuous Random Variables
}

\author{
Ying-ying Zhang \\ Department of Statistics and Actuarial Science, Chongqing University, Chongqing, China
}

\begin{abstract}
First of all, we derive theoretically a calculation formula of one-sided and two-sided $P$ value for the general one-dimensional continuous random variables. Then, we give two examples to illustrate the application of this formula. Example 1 considers $\chi^{2}(d f)$. Example 2 considers $Z \sim N(0,1)$, in this example, we make a further discussion on the two-sided $\mathbf{P}$ value calculation, and also point out a common mistake in the real calculation of the $\mathbf{P}$ value. Finally, we hope the theoretical derivation can clarify some confusions in the calculation of the $P$ value.
\end{abstract}

Keywords—P value, $P_{-}$_value() function, hypothesis testing, continuous random variables

\section{一维连续型随机变量的单侧和双侧 P 值计算公式的理 论推导}

张应应

重庆大学, 重庆, 中国

\begin{abstract}
摘 要 首先, 我们对一般的一维连续型随机变量从理论上推导了单侧和双侧 $\mathrm{P}$ 值的计算公式。然后, 我们给出了两个例子来说 明此计算公式的应用。例 1 考虑的是 $\chi^{2}(d f)$ 。例 2 考虑的是 $Z \sim N(0,1)$, 在此例中, 我们对双侧 $\mathrm{P}$ 值的计算进行了进一步的讨论, 还指出了实际 $\mathrm{P}$ 值计算中常犯的一个错误。最后，我们希望本理论推导可以澄清在 P 值计算中的一些困惑。
\end{abstract}

关键词 P 值, P_value()函数, 假设检验, 连续型随机变量

\section{1. 引言}

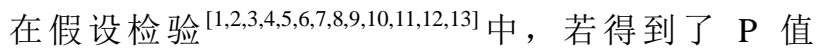
$[14,15,16,17,18,19,20]$, 则检验标准为: 当 $\mathrm{P}$ 值小于指定的显著性 水平 $\alpha$ 时, 则拒绝原假设; 否则不拒绝原假设。因此 $\mathrm{P}$ 值 的计算非常重要。[9]中已有一个 R 函数 P_value()可以计算 一维任意的连续型随机变量的单侧或双侧 $\mathrm{P}$ 值。但是[9]中 关于 $\mathrm{P}$ 值的理论推导比较简单, 并且只是针对 $Z \sim N(0,1)$ 的 情形推导了, 并未对一般的一维连续型随机变量的 $\mathrm{P}$ 值计 算进行推导。本文对一般的一维连续型随机变量的 P 值计 算进行了理论推导。我们希望本理论推导可以澄清 $\mathrm{P}$ 值计 算的一些困惑。

本文剩余部分安排如下: 第 2 部分推导一般的一维连
续型随机变量的 $\mathrm{P}$ 值计算公式, 并给出两个例子来说明其 应用。第 3 部分总结。

\section{2. 主要结果}

我们首先对一般的一维连续型随机变量推导单侧和双 侧 $\mathrm{P}$ 值的计算公式。假设连续型随机变量 $X$ 的 $\mathrm{pdf}$ 是 $f_{X}(x), \operatorname{cdf}$ 是 $F_{X}(x)$, 支集是 $X$ 。

注 1. $X$ 可以是 $(-\infty, \infty)$, 比如 $N\left(\mu, \sigma^{2}\right), t(d f)$, DoubleExponential $(\mu, \sigma), \operatorname{Logistic}(\mu, \beta), X$ 也可以是 $(0, \infty)$, 比如 $\chi^{2}(d f), F\left(d f_{1}, d f_{2}\right), \operatorname{Gamma}(\alpha, \beta), \operatorname{Exponential}(\beta)$, $\operatorname{Lognormal}\left(\mu, \sigma^{2}\right), \operatorname{Weibull}(\gamma, \beta), X$ 还可以是 $(a, b)$, 比如 $\operatorname{Uniform}(a, b), \operatorname{Beta}(\alpha, \beta)(a=0, b=1)$ 等。 
此文采用上分位数, 即 $P\left(X \geq X_{\alpha}\right)=\alpha$, 其中 $X_{\alpha}=F_{X}^{-1}(1-\alpha)$ 是 $X$ 的上 $\alpha$ 分位数, 因为

$$
F_{X}\left(X_{\alpha}\right)=P\left(X \leq X_{\alpha}\right)=1-P\left(X \geq X_{\alpha}\right)=1-\alpha .
$$

我们先考虑双侧假设的情形。

$$
H_{0}: \theta=\theta_{0}, \quad H_{1}: \theta \neq \theta_{0} .
$$

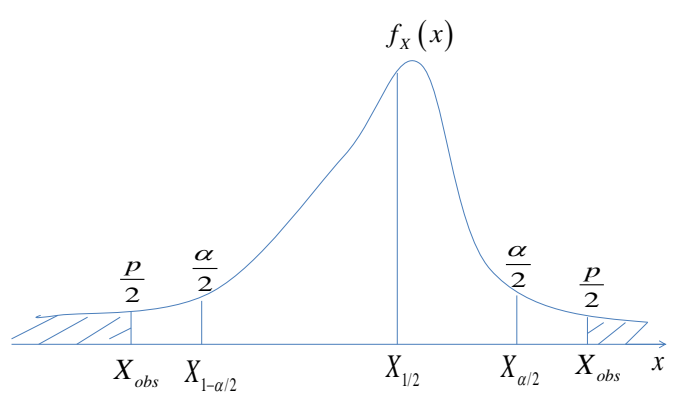

图 1. 双侧假设时 $X$ 的密度函数和分位数图

双侧假设时 $X$ 的密度函数和分位数图见图 1。此时对 应着 side $=0$, 拒绝域为

$$
R=\left\{\boldsymbol{x}: X_{o b s}<X_{1-\alpha / 2} \text { or } X_{o b s}>X_{\alpha / 2}\right\} .
$$

此时的 $\mathrm{P}$ 值是 $F_{X}\left(X_{o b s}\right)=P\left(X \leq X_{o b s}\right)$ 的分段函数。对照 图 1, 当 $X_{o b s}<X_{1 / 2}$, 即 $F_{X}\left(X_{o b s}\right)<F_{X}\left(X_{1 / 2}\right)=\frac{1}{2}$ 时,

$$
\text { 拒绝 } H_{0} \Leftrightarrow X_{o b s}<X_{1-\alpha / 2} \Leftrightarrow \frac{p}{2}<\frac{\alpha}{2} \Leftrightarrow p<\alpha .
$$

从而 $\mathrm{P}$ 值的定义应该满足 $\frac{p}{2}=P\left(X \leq X_{o b s}\right)$, 因此

$$
p=2 P\left(X \leq X_{o b s}\right)=2 F_{X}\left(X_{o b s}\right) .
$$

当 $X_{o b s} \geq X_{1 / 2}$, 即 $F_{X}\left(X_{o b s}\right) \geq F_{X}\left(X_{1 / 2}\right)=\frac{1}{2}$ 时,

$$
\text { 拒绝 } H_{0} \Leftrightarrow X_{o b s}>X_{\alpha / 2} \Leftrightarrow \frac{p}{2}<\frac{\alpha}{2} \Leftrightarrow p<\alpha .
$$

从而 $\mathrm{P}$ 值的定义应该满足 $\frac{p}{2}=P\left(X \geq X_{o b s}\right)$, 因此 $p=2 P\left(X \geq X_{o b s}\right)=2\left[1-P\left(X \leq X_{o b s}\right)\right]=2\left[1-F_{X}\left(X_{o b s}\right)\right]$.

综上, 我们得到在双侧假设的情形下, $\mathrm{P}$ 值的定义为

$$
p=\left\{\begin{array}{cc}
2 F_{X}\left(X_{o b s}\right), & \text { 若 } F_{X}\left(X_{o b s}\right)<\frac{1}{2}, \\
2\left[1-F_{X}\left(X_{o b s}\right)\right] & \text { 若 } F_{X}\left(X_{o b s}\right) \geq \frac{1}{2} .
\end{array}\right.
$$

现在我们再来考虑单侧假设的情形。分两种情形讨论。 (a) $H_{0}: \theta \geq \theta_{0}, \quad H_{1}: \theta<\theta_{0}$.

单侧假设 $\left(H_{1}: \theta<\theta_{0}\right)$ 时 $X$ 的密度函数和分位数图见 图 2。此时对应着 side $<0$ （与备择假设的不等号的方向相 同), 拒绝域为

$$
R=\left\{\boldsymbol{x}: X_{o b s} \leq X_{1-\alpha}\right\} .
$$

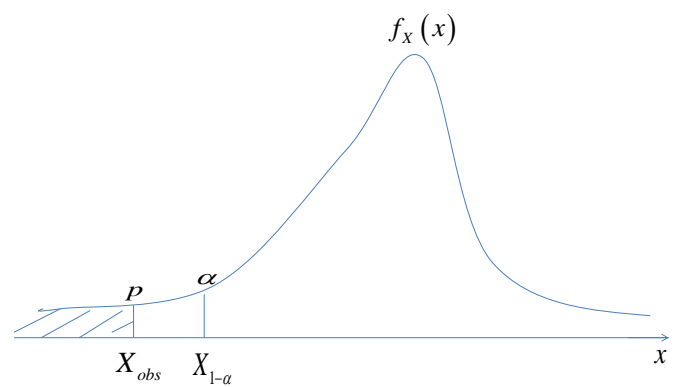

图 2. 单侧假设 $\left(H_{1}: \theta<\theta_{0}\right)$ 时 $X$ 的密度函数和分位数图

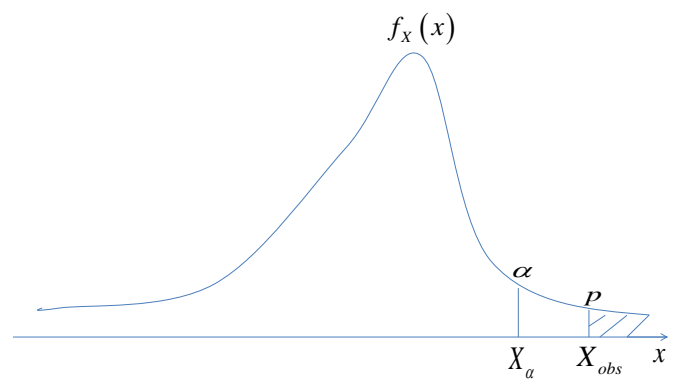

图 3. 单侧假设 $\left(H_{1}: \theta>\theta_{0}\right)$ 时 $X$ 的密度函数和分位数图

对照图 2, 我们有

$$
\text { 拒绝 } H_{0} \Leftrightarrow X_{o b s} \leq X_{1-\alpha} \Leftrightarrow p<\alpha .
$$

从而 $\mathrm{P}$ 值的定义应该满足

$$
p=P\left(X \leq X_{o b s}\right)=F_{X}\left(X_{o b s}\right) .
$$

(b) $H_{0}: \theta \leq \theta_{0}, \quad H_{1}: \theta>\theta_{0}$.

单侧假设 $\left(H_{1}: \theta>\theta_{0}\right)$ 时 $X$ 的密度函数和分位数图见 图 3。此时对应着 side $>0$ (与备择假设的不等号的方向相 同), 拒绝域为

$$
R=\left\{\boldsymbol{x}: X_{o b s} \geq X_{\alpha}\right\} .
$$

对照图 3, 我们有

$$
\text { 拒绝 } H_{0} \Leftrightarrow X_{o b s}>X_{\alpha} \Leftrightarrow p<\alpha .
$$

从而 $\mathrm{P}$ 值的定义应该满足

$$
p=P\left(X \geq X_{o b s}\right)=1-P\left(X \leq X_{o b s}\right)=1-F_{X}\left(X_{o b s}\right) .
$$



为

综合单侧和双侧假设的讨论, 可归纳得到 P 值的定义

$$
p= \begin{cases}F_{X}\left(X_{o b s}\right), & \text { 若side }<0, \\ 1-F_{X}\left(X_{o b s}\right), & \text { 若side }>0, \\ 2 F_{X}\left(X_{o b s}\right), & \text { 若side }=0 \text { 且 } F_{X}\left(X_{o b s}\right)<1 / 2, \\ 2\left[1-F_{X}\left(X_{o b s}\right)\right], & \text { 若 side }=0 \text { 且 } F_{X}\left(X_{o b s}\right) \geq 1 / 2 .\end{cases}
$$

由(2)式, 我们可以编写一个 $\mathrm{R}$ 函数来求任意的连续型 随机变量 $X$ 的 $\mathrm{P}$ 值。这个工作已经完成了, 见[9]中的 P_value()函数。举两个特殊的例子来说明(2)的应用。

例1. $X \sim \chi^{2}(d f)$

注意此例中 $X=(0, \infty)$, 此分布也可以换成其它的 $X=(0, \infty)$ 的分布, 见注 1 。例 1 的推导只需要照搬前面的 对一般的一维连续型随机变量 $X$ 的推导即可, 只需把 $X$ 换 成 $\chi^{2}(d f)$ 。

例2. $X=Z \sim N(0,1)$

注意此例 中 $X=(-\infty, \infty)$, 且 它是关于 0 点 $\left(0=F_{Z}^{-1}(1 / 2)=Z_{1 / 2}\right)$ 对称的分布。此分布也可以换成其它 的 $X=(-\infty, \infty)$ 的分布, 见注 1 。例 2 中关于单侧假设的推 导也可以照搬前面的对一般的一维连续型随机变量 $X$ 的推 导，只需把 $X$ 换成 $Z$ 即可。下面讨论 $Z$ 的双侧假设的情形。

$$
H_{0}: \theta=\theta_{0}, \quad H_{1}: \theta \neq \theta_{0} \text {. }
$$

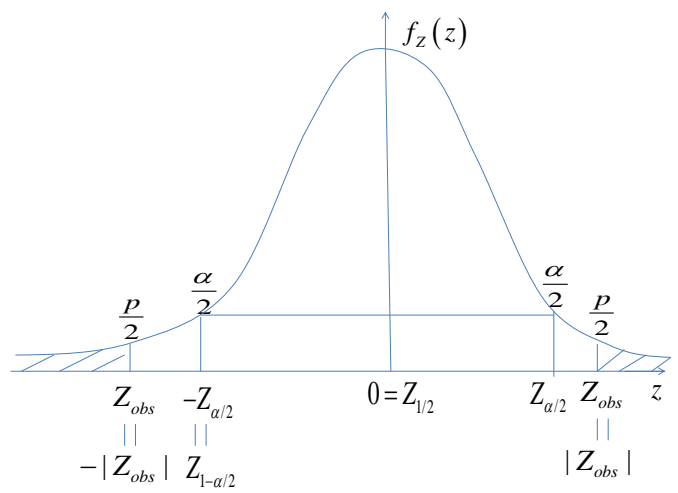

图 4. 双侧假设时 $Z$ 的密度函数和分位数图

双侧假设时 $Z$ 的密度函数和分位数图见图 4。此时对 应着 $\operatorname{side}=0$, 拒绝域为

$$
R=\left\{\boldsymbol{x}:\left|Z_{o b s}\right|>Z_{\alpha / 2}\right\} .
$$

在图 4 中, 由对称性有 $Z_{1-\alpha / 2}=-Z_{\alpha / 2}$ 。对照图 4, 我们 有

$$
\text { 拒绝 } H_{0} \Leftrightarrow\left|Z_{o b s}\right|>Z_{\alpha / 2} \Leftrightarrow \frac{p}{2}<\frac{\alpha}{2} \Leftrightarrow p<\alpha \text {. }
$$

从而 $\mathrm{P}$ 值的定义应该满足

$$
\frac{p}{2}=P\left(Z \geq\left|Z_{o b s}\right|\right)=P\left(Z \leq-\left|Z_{o b s}\right|\right) .
$$

在上式中 $Z \sim N(0,1)$ 是一个随机变量, $Z_{o b s}$ 是 $Z$ 的实现 值, 它可正可负。因此

$$
\begin{aligned}
p & =2 P\left(Z \geq\left|Z_{o b s}\right|\right)=2 P\left(Z \leq-\left|Z_{o b s}\right|\right) \\
& =P\left(Z \geq\left|Z_{o b s}\right|\right)+P\left(Z \leq-\left|Z_{o b s}\right|\right) \text { (由对称性) } \\
& =P\left(|Z| \geq\left|Z_{o b s}\right|\right) .
\end{aligned}
$$

注意(3)与 (1) 是等价的。在 $N(0,1)$ 或类似的关于对称轴 对称的分布中, $\mathrm{P}$ 值的计算一般采用类似于(3)的带 $\left|Z_{o b s}\right|$ 的 公式, 却忽略了像(1)的不带 $\left|Z_{o b s}\right|$ 的分段函数的公式。现在 我们来证明 (3)与 (1) 的等价性。当 $Z_{o b s}<Z_{1 / 2}=0$, 即 $F_{Z}\left(Z_{o b}\right)<F\left(Z_{2}\right) \frac{1}{2}$ 时, 由(3)得

$$
\begin{aligned}
p & =P\left(|Z| \geq\left|Z_{o b s}\right|\right)=2 P\left(Z \leq-\left|Z_{o b s}\right|\right) \\
& =2 P\left(Z \leq Z_{o b s}\right)=2 F_{Z}\left(Z_{o b s}\right)
\end{aligned}
$$

当 $Z_{o b s} \geq Z_{1 / 2}=0$, 即 $F_{Z}\left(Z_{o b s}\right) \geq F_{Z}\left(Z_{1 / 2}\right)=\frac{1}{2}$ 时, 由(3)得

$$
\begin{aligned}
p & =P\left(|Z| \geq\left|Z_{o b s}\right|\right)=2 P\left(Z \geq\left|Z_{o b s}\right|\right)=2 P\left(Z \geq Z_{o b s}\right) \\
& =2\left[1-P\left(Z \leq Z_{o b s}\right)\right]=2\left[1-F_{Z}\left(Z_{o b s}\right)\right] .
\end{aligned}
$$

从而(3)与(1)是等价的。

(3)中的 $\mathrm{P}$ 值可以用 $Z$ 的分布函数 $F_{Z}(\cdot)$ 来表示:

$$
\begin{aligned}
p & =P\left(|Z| \geq\left|Z_{o b s}\right|\right)=2 P\left(Z \geq\left|Z_{o b s}\right|\right) \\
& =2\left[1-P\left(Z \leq\left|Z_{o b s}\right|\right)\right]=2\left[1-F_{Z}\left(\left|Z_{o b s}\right|\right)\right] .
\end{aligned}
$$

在实际计算 $\mathrm{P}$ 值中, 人们一般采用类似于(3)的带 $\left|Z_{o b s}\right|$ 的公式, 但是很容易出错, 比如漏掉了 2 倍, 或者把 $|Z|$ 的 绝对值写掉了, 写成

$$
p=P\left(Z \geq\left|Z_{o b s}\right|\right) .
$$

比如在一元线性回归的 summary(lm.sol) 中, Coefficients 部分会报道 $P$ 值 $=\operatorname{Pr}(>|t|)$, 此时的 $\mathrm{P}$ 值应为

$$
\begin{aligned}
p & =P\left(|T| \geq\left|T_{o b s}\right|\right)=2 P\left(T \geq\left|T_{o b s}\right|\right)=2\left[1-P\left(T \leq\left|T_{o b s}\right|\right)\right] \\
& =2\left[1-F_{T}\left(\left|T_{o b s}\right|\right)\right]=2\left[1-\operatorname{pt}\left(\left|T_{o b s}\right|, d f=n-2\right)\right],
\end{aligned}
$$

其中 $T \sim t(n-2), T_{o b s}$ 是 $T$ 的观测值。

\section{3. 总结}

首先, 我们对一般的一维连续型随机变量从理论上推 
导了单侧和双侧 $\mathrm{P}$ 值的计算公式(2)。然后, 我们给出了两 个例子来说明(2)的应用。例 1 考虑的是 $\chi^{2}(d f)$, 它的 $\mathrm{P}$ 值 推导只需要照搬一般的一维连续型随机变量的推导即可。 例 2 考虑的是 $Z \sim N(0,1)$, 在此例中, 我们对双侧 $\mathrm{P}$ 值的计 算进行了进一步的讨论, 给出了一般情形下采用的公式(3), 并证明了(3)和 $F_{X}\left(X_{o b s}\right)$ 的分段函数(1)的等价性, 还指出了 实际 $\mathrm{P}$ 值计算中常犯的一个错误。最后, 我们希望本理论 推导可以澄清在 $\mathrm{P}$ 值计算中的一些困惑。

\section{参考文献(References)}

[1] D. Freedman et al. (authors), Z.S. Wei et al. (translators), Statistics. Beijing: China Statistics Press, 1997.

[2] R.A. Johnson and D.W. Wichern (authors), X. Lu (translator). Applied Multivariate Statistical Analysis. Fourth Edition. Beijing: Tsinghua University Press, 2001.

[3] H. Yang, Q.S. Liu, and B. Zhong, Mathematical Statistics. Beijing: Higher Education Press, 2004.

[4] H.X. Gao, Applied Multivariate Statistical Analysis. Beijing: Peking University Press, 2005.

[5] H. Yang, B. Zhong, and Q.S. Liu, Applied Mathematical Statistics. Beijing: Tsinghua University Press, 2006.

[6] Y.C. Tang, $R$ Language and Statistical Analysis. Beijing: Higher Education Press, 2008.

[7] X.R. Chen, G.X. Ni, and C.S. Chen, Mathematical Statistics Course. Hefei: Press of University of Science and Technology of China, 2009.

[8] X.M. Wang, Applied Multivariate Analysis. Third Edition. Shanghai: Shanghai University of Finance and Economics
Press, 2009.

[9] Y. Xue and L.P. Chen, Statistical Modeling and R Software. Beijing: Tsinghua University Press, 2009.

[10] R.C. Zhang, Mathematical Statistics. Beijing: Science Press, 2010.

[11] X.S. Ren and X.L. Yu, Multivariate Statistical Analysis. Second Edition. Beijing: China Statistics Press, 2011.

[12] X.Q. He, Multivariate Statistical Analysis. Third Edition. Beijing: China Renmin University Press, 2012.

[13] S.L. Li, Data Analysis and R Software. Beijing: Science Press, 2013.

[14] D.M. Fan, "The P value of hypothesis test," Journal of Zhengzhou Economic Management Cadre Institute, vol. 17, no. 4, pp. 70-71, 2002.

[15] Z.X. Han and L. Zhang, "P value test and hypothesis testing," The Border Economy and Culture, no. 4, pp. 62-63, 2006.

[16] J.H. Fu, "Classic test P values on several issues," Statistics and Decision, no. 1, pp. 156-157, 2009.

[17] J.K. Chen, "On the P value of testing statistical hypotheses," Value Engineering, no. 25, pp. 257-258, 2011.

[18] G.Z. Zhi et al., "The flexible application of value $P$ in hypothesis tests," College Mathematics, vol. 27, no. 5, pp. 152-156, 2011.

[19] M.H. Zhang, "Information entropy and P value," Mathematical Medicine Magazine, vol. 25, no. 2, pp. 189-191, 2012.

[20] G. Yang, "The study of P value in the hypothesis testing," Journal of Henan Institute of Engineering (Natural Science Edition), vol. 24, no. 2, pp. 65-67, 2012. 